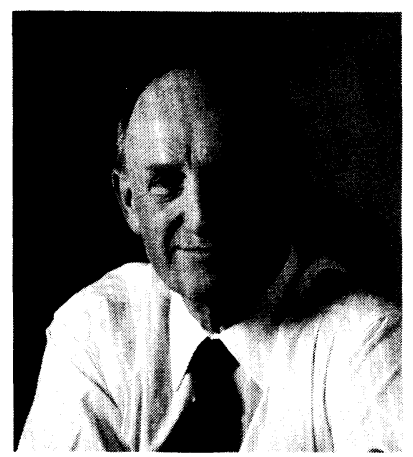

\section{Celebration and Recognition}

It has now become a tradition of several years standing to acknowledge the winners of the Kingslake Medal and Prize in the August issue of Optical Engineering. This acknowledgement comes at a time when many of us have been through a variety of celebrations and recognitions of achievement. Those of us in academic life or with family members graduating this year will have attended Commencements, Degree Days, Degree Convocations, etc. during the last two months - at least those of us in the northern hemisphere. For me, personally, it has been a very special year. I attended my last set of commencement exercises at the University of Rochester as its Provost. I look forward to attending future ceremonies without having to perform! My last official ceremony as Provost of the University of Rochester was a rather personal one to attend and participate in: the Degree Congregation at the Lancaster University in England where my brother, Geoffrey, received an honorary degree of Doctor of Laws (Honoris Causa). It was with a sense of great pride that I processed with him and other dignitaries and met the University's Chancellor, Her Royal Highness Princess Alexandra, who presented the degrees. A fine ceremony and a wonderful day for our family.

\section{The Kingslake Medal and Prize}

It is a great pleasure to congratulate the winners of the Kingslake Medal and Prize for the best article to appear in Optical Engineering during the calendar year 1993 (Volume 32). The winners selected by a distinguished international selection committee chaired by Dr. Satoshi Ishihara were Harvey M. Phillips and Roland A. Sauerbrey of Rice University for their paper entitled "Excimer-laser-produced nanostructures in polymers" that appeared in the October issue [Vol. 32(10), 2424-2436].

As the chair of the selection committee noted, "the selection task this year was harder than last year ... because the number of papers increased by $32.2 \%$...." He goes on to say that "we started by picking 90 papers as potential candidates from the entire 423 papers in Volume 32 . In the second round we concentrated our attention mainly on 11 papers and in the third squeezed out 3 papers. Anyway, I am pleased to say that the final result was reached unanimously again this year."

The selection committee of Jean Bulabois, John Greivenkamp, Bruce Steiner, John Walkup, and Satoshi Ishihara did a great job and deserves our thanks.

Brian J. Thompson Editor

\begin{tabular}{|c|c|c|c|}
\hline \multicolumn{4}{|c|}{ Rudolf Kingslake Medal and Prize-Past Recipients } \\
\hline 1974 & Irving R. Abel and B. R. Reynolds & 1985 & Armand R. Tanguay, Jr. \\
\hline 1975 & J. M. Burch and C. Forno & 1986 & Arthur D. Fisher, Lai-Chang Ling, John N. Lee, \\
\hline 1976 & Richard E. Swing & & and Robert C. Fukuda \\
\hline 1977 & David B. Kay and Brian J. Thompson & 1987 & Chris P. Kirk \\
\hline 1978 & Norman J. Brown & 1988 & Ares J. Rosakis, Alan T. Zehnder, and \\
\hline 1979 & J. R. Fienup & & Ramaratnam Narasimhan \\
\hline 1980 & G. Ferrano and G. Hausler & 1989 & Pochi Yeh, Arthur Chiou, John Hong, Paul H. \\
\hline 1981 & Robert A. Sprague and William D. Turner & & Beckwith, Tallis Chang, and Monte Khoshnevisan \\
\hline 1982 & David M. Pepper & 1990 & Paul R. Prucnal and Philippe A. Perrier \\
\hline 1983 & James R. Palmer & 1991 & Brian E. Newnam \\
\hline 1984 & Gene R. Gindi and Arthur F. Gmitro & 1992 & Aden B. Meinel and Marjorie P. Meinel \\
\hline
\end{tabular}




September 1994
Optics in South Africa
Hannes Markusse
ELOPTRO
Institute of Atomic Physics
P.O. Box 869
Kempton Park 1620, South Africa
Maurice W. McDowell
CSIR/Production Technology Div.
Productiontek
P.O. Box 395
Pretoria 0001, South Africa
27 12 841 3418 -27 12 841 2131 FAX
October 1994
Optics in Russia
V. Ya. Panchenko
Scientific Research Center for Technological
Lasers
Russia Academy of Sciences
B-333, Gubkina, 3
117971 Moscow, Russia
E-mail: ilc@ compnet.npimsu.msk.su
(095)135-54-30 095$) 334-02-01$ FAX
Digital Image Recovery and Synthesis
Paul S. Idell
Rockwell International
Rocketdyne Division
MS SS46
P.O. Box 7922
Canoga Park, CA 91309-7922
818/586-8238 • 818/586-5295 FAX
E-mail: pidell@ windance.rdyne.rockwell.com

November 1994

Micro-Optics

Chandrasekhar Roychoudhuri

University of Connecticut at Storrs

Photonics Research Center

MS-157, Room 312

260 Glenbrook Road

Storrs, CT 06269-3157

203/486-4816 • 203/486-3789 FAX

\section{December 1994}

Optics in Ireland

John Hegarty

University of Dublin

Trinity College

Department of Pure and Applied Physics

Dublin 2, Ireland

+353-1-7022019 • +353-1-711759 FAX

Conleth D. Hussey

University of Limerick

Lightwave Technology Research Ctr.

Plassey Pk.

Limerick, Ireland

35261333644 • 35261330316 FAX

February 1995

High Heat Flux Optical Engineering

Ali M. Khounsary

Argonne National Laboratory

Advanced Photon Source, APS 362

Argonne, IL 60439

$708 / 252-3384 \cdot 708 / 252-3222 \mathrm{FAX}$

\author{
March 1995 \\ Optical Engineering in Ophthalmology \\ Suganda Jutamulia \\ Kowa Company, Ltd. \\ Silicon Valley Office \\ 100 Homeland Court, Suite 302 \\ San Jose, CA 95112 \\ 408/441-9300 • 408/441-0537 FAX \\ Toshimitsu Asakura \\ Hokkaido University \\ Research Institute for Electronic Science \\ Sapporo, 060 Japan \\ 81-11-716-2111 • 81-11-758-3173 FAX \\ April 1995

\section{Optics in the Ukraine} \\ Oleg V. Angelsky \\ Chernovtsy University \\ Department of Correlation Optics \\ 2 Kosyubinsky Street \\ 274012 Chernovtsy \\ Ukraine \\ (03722) 44730 • (03722) $41314 \mathrm{FAX}$
}

May 1995

X-Ray/EUV Optics

Richard B. Hoover

NASA Marshall Space Flight Center

ES52 Space Science Center

Huntsville, AL 35812-0001

E-mail: hoover@ssl.msfc.nasa.gov

205/544-7617 • 205/544-5856 FAX

July 1995

Optics in Switzerland

P. K. Rastogi

Swiss Federal Institute of Technology-Lausanne

Laboratory of Stress Analysis

CH-1015 Lausanne

Switzerland

E-mail: rastogi@elgc.epfl.ch

(021) 6932445 • (021) 6934748 FAX

Manuscripts due Nov. 15, 1994

September 1995

Optical Science \& Engineering in Finland

Seppo Honkanen

Nokia Research Center

P.O. Box 45

FIN-00211 Helsinki

Finland

+35804376471 •+35804552557 FAX

Manuscripts due Jan. 15, 1995

November 1995

Optical Remote Sensing and Image

Processing

Mohammad A. Karim

Bradley D. Duncan

University of Dayton

Center for Electro-Optics

300 College Park

Dayton, OH 45469-0227

$513 / 229-2241 \cdot 513 / 229-3177$

513/229-2471 FAX

Manuscripts due April 1, 1995

\section{December 1995}

Optics in Polymer Science and Technology

Maksymilian Pluta

Institute of Applied Optics

Kamionkowska 18

03-805 Warsaw, Poland

(4822) $184405 \cdot$ (4822) 133265 FAX

Andrzej Wasiak

Institute of Fundamental Technological

Research

Polish Academy of Sciences

Savietokrzyska 21

00-012 Warsaw, Poland

(4822) 269815 FAX

Manuscripts due April 1, 1995.

January 1996

Optical Science and Engineering in Argentina

Guillermo H. Kaufmann

Universidad Nacional de Rosario

Instituto de Fisica Rosario

Applied Optics Group

Bv. 27 de Febrero 210 bis

2000 Rosario

Argentina

E-mail: guille@ifir.edu.ar

or gkaufman@arosario.bitnet

$5441825838 \cdot 5441257164$ FAX

Manuscripts due June 1, 1995

Visual Communications and Image Processing

Cheng-Tie Chen

Bellcore, NVC 3X-321

331 Newman Springs Road

Red Bank, NJ 07701

908/758-3106 • 908/758-4371 FAX

E-mail: ctc@nyquist.bellcore.com

Kou-Hu Tzou

COMSAT Laboratories

22300 Comsat Drive

Clarksburg, MD 20871

301/428-4663 - 301/428-9287 FAX

E-mail: kouhu@ctd.comsat.com

Ya-Qin Zhang

GTE Laboratories

40 Sylvan Road

Waltham, MA 02254

617/466-2259 • 617/890-9320 FAX

E-mail: yz00@gte.com

Manuscripts due June 1, 1995

\section{February 1996}

Optical Engineering in Small Companies

Xiangyang Yang

Quantex Corporation

2 Research Court

Rockville, MD 20850

301/258-2701 • 301/258-9871 FAX

William J. Miceli

Office of Naval Research

800 North Quincy Street

Arlington, VA $22217-5000$

703/696-5752 - 703/696-1330 FAX

Manuscripts due July 1, 1995 